\title{
STUDIES ON OXYTETRACYCLINE RESIDUES DEPLETION IN RABBIT MEAT
}

\author{
MORSHDY, A.M. ; HUSSEIN, M.A. ${ }^{*}$ and EL-GOHARY, A.E. ${ }^{* *}$ \\ ${ }_{*}^{*}$ Food Control Dept.-Faculty of Vet. Medicine-Zagazig, University-Zagazig, Egypt. \\ ${ }^{* * *}$ Food Control Lab. -Dammietta Seaport-Animal Health Research Institute, Egypt. \\ Email: elged2010@yahoo.com
}

\section{ABSTRACT}

Received at: 3/2/2014

A total of 100 rabbits weighed about 1000-1500 grams (free from any antibiotics by feeding the rabbits on a balanced ration free from antibiotics for three weeks) were divided into two groups ; control group (50) and test group (50) which were injected subcutaneously with oxytetracycline hydrochloride $20 \mathrm{mg} / \mathrm{kg}$ body weight for five

Accepted: 2/6/2014 successive days. 5 rabbits from each group were slaughtered at zero time, $24 \mathrm{hr}, 48 \mathrm{hr}$, $72 \mathrm{hr}, 96 \mathrm{hr}, 120 \mathrm{hr}, 144 \mathrm{hr}, 168 \mathrm{hr}$ (1week), 2weeks and 3weeks. Residue depletion of oxytetracycline was determined using high performance liquid chromatography (HPLC). The slaughter time strongly affects the concentration of oxytetracycline residues in animal meats, so that by increasing the slaughter time the oxytetracycline residues decreased. Boiling for 30 minutes caused a great degradation of oxytetracyline residues in rabbit meats but not complete destruction. Freezing at $-20^{\circ} \mathrm{c}$ caused a lower degradation than that caused by boiling. So neither boiling nor freezing could be used as reliable methods to get rid of oxytetracycline residues in rabbit meats. The recovery rates for oxytetracycline in rabbit muscles, kidneys and livers were $102 \%, 92 \%$ and $86 \%$, respectively, at a concentration of $20 \mu \mathrm{g} / \mathrm{kg}$ of spiked samples.

Key words: Oxytetracycline, Residues, Rabbit meat.

\section{INTRODUCTION}

Rabbit meat is considered a high quality product due to its high protein and low saturated fatty acids, cholesterol and sodium content. Antibiotics are widely used in veterinary medicine and subsequently drug residues may persist in foods derived from animals, which may pose an adverse health effect for the consumer. Today antimicrobial drugs are used to control, prevent and treat infection, and to enhance animal growth and feed efficiency. Tetracyclines are most widely used antibiotics in veterinary medicine in Egypt due to its broad spectrum of antimicrobial activity, availability and low cost. Violation of the maximum residue limit (MRL) regulation can only be proven with fully validated chromatographic methods such as high performance liquid chromatography (HPLC) (Moats, 1997). The use of antimicrobials in food-producing animals may result in the presence of residues in foodstuffs of animal origin. Protection of public health against possible harmful effects of veterinary drug residues is a relatively recent preoccupation. The initial intention for adequate consumer protection led to the desire to achieve a complete elimination of all traces of drug residues in food commodities. Therefore, animal drugs were initially approved based on a "no residue" tolerance policy, but actually the "zero" tolerance represented the sensitivity of the analytical method used to monitor for drug residues. As analytical methods improved, the "no residue" tolerance was continually being lowered. Ultimately, a policy of negligible tolerance, based on toxicology data, was developed (Boisseau, 1993; Teske, 1993). The legislation for pharmaceutical and veterinary products established a withdrawal period of antibiotics of 28 days for fattening rabbits, limiting the addition of antibiotics to the first days of fattening (Badiola et al., 2007). Since the most of foods-producing animals are always cooked before consumption and the variations in oxytetracycline levels in the meat are dependent on type of cooking. More findings about the effect of cooking on oxytetracycline residue are needed to accurately determine consumer exposure to this drug. The boiling for 30 minutes and roasting at $150^{\circ} \mathrm{C}$ for 30 minutes caused a complete degradation of drug residues. Freezing at $-20^{\circ} \mathrm{C}$ ensured gradual degradation of the residues remained in different meats of the medicated rabbits (Gehad, 2002). The acceptable MRLs for tetracyclines as recommended by the Joint FAO/WHO Expert Committee on Food Additives is 200, 600, and $1200 \mu \mathrm{g} / \mathrm{kg}$ for muscles, liver, and kidney, respectively, (Mehran et al., 2009). The abundant and improper use of tetracycline antibiotics may result in the presence of their residues in edible animal meats, which can be toxic and dangerous for human health and potentially cause allergic reactions. In addition, low-level doses of antibiotic in foodstuffs consumed for long periods can lead to the spread of drug-resistant microorganisms (Yu et al., 2011). Therefore, this work was designed to determine the residue depletion of oxytetracycline 
in rabbit meats and the effect of boiling and freezing on oxytetracycline residues.

\section{MATERIALS and METHODS}

\section{Materials:}

1- HPLC grade acetonitrile, methanol and water.

2- AR/GR grade disodium hydrogen phosphate (Na2HPO4), citric acid and oxalic acid.

3- Oasis HLB cartridge $6 \mathrm{~cm} 3$ (200 mg).

4- From pure standard of oxytetracyline (assay 96.2\% in HPLC), as their hydrochloride, individual stock standard solution at $1 \mathrm{mg} / \mathrm{mL}$ (free base) was prepared in methanol in an amber color volumetric flask and was stored at $-20^{\circ} \mathrm{C}$ in the dark for maximum period of 2 months. A composite working standard solutions of $300,250,200,160,80,40$ and $20 \mu \mathrm{g} / \mathrm{mL}$ were prepared by diluting stock solution with methanol. As it is unstable at room temperature, so prepared daily and stored at $4{ }^{\circ} \mathrm{C}$. For analysis, $0.01 \mathrm{M}$ oxalic acid ( $\mathrm{pH}$ 1.6), $0.1 \mathrm{M}$ citric acid and $0.2 \mathrm{M}$ disodium hydrogen phosphate (Na2HPO4) buffer were prepared in Milli-Q water and filtered through 0.22 $\mu \mathrm{m}$ cellulose filter.

5- $0.01 \mathrm{M}$ methanolic oxalic acid ( $\mathrm{pH}$ 1.86) was prepared in methanol.

6- McIIvaine buffer ( $\mathrm{pH}$ 3.85) was prepared by mixing $278 \mathrm{~mL}$ of $0.1 \mathrm{M}$ citric acid solution in 222 $\mathrm{mL}$ of $0.2 \mathrm{M} \mathrm{Na} 2 \mathrm{HPO} 4$ solution and the $\mathrm{pH}$ was adjusted to 3.85 with extra citric acid solution. All these buffer solutions were stored at $4^{\circ} \mathrm{C}$.

\section{Methods:}

\section{Injection of rabbits with oxytetracyclines:}

Hundred rabbits weighed about 1000-1500 grams(free from any antibiotics by feeding the rabbits on a balanced ration free from antibiotics for three weeks) were divided into two groups; control group (50) and test group (50) which were injected subcutaneously with oxytetracycline hydrochloride $20 \mathrm{mg} / \mathrm{kg}$ body weight for five successive days.

\section{Slaughtering of rabbits:}

Five rabbits from each group were slaughtered at zero time, 24 hr, 48 hr, 72 hr, 96 hr, $120 \mathrm{hr}, 144 \mathrm{hr}, 168 \mathrm{hr}$ (1 week), 2 weeks and 3 weeks.

\section{Effect of boiling and freezing on oxytetracycline residues in rabbit meats.}

The samples were taken from meat, liver and kidney directly after slaughtering and were divided as follow:

1st group: Consists of 100 samples from each type (meat, liver and kidney).The oxytetracycline residues were determined using HPLC without treatment (boiling or freezing). 2nd group: Consists of 100 samples from each type (meat, liver and kidney). The oxytetracycline residues were determined using HPLC after boiling for 30 $\min$.

3rd group: Consists of 100 samples from each type (meat, liver and kidney). The oxytetracycline residues were determined using HPLC after freezing at $-20^{\circ} \mathrm{C}$ for one, two and three months.

\section{Determination of oxytetracycline residues by HPLC method:-}

The method recommended by Biswas et al. (2007) was used.

\subsection{Instrumentation:}

HPLC and uv detector. The analytical column was a Luna $5 \mu \mathrm{C} 8$ (RP- C8) column $(4.6 \times 250 \mathrm{~mm}, 5 \mu \mathrm{m}$ particle size). The optimized mobile phase for desorption and separation was a mixture of $0.01 \mathrm{M}$ oxalic acid/acetonitrile/methanol (77:18:5, v/v/v), and the flow rate was $0.6 \mathrm{~mL} / \mathrm{min}$. The detection was performed at $355 \mathrm{~nm}$ with scanning range $340-360$ nm.

\subsection{Method of extraction:}

1- Frozen meat samples were thawed and finely diced with scissors after trimming off external fat and fascia.

2- The finely cut samples were blended in a high speed $(15,000 \mathrm{rpm})$ meat blender for $2 \mathrm{~min}$.

3- A representative portion of this sample (10 g) was weighed into a polypropylene tube and homogenized with $10 \mathrm{~mL}$ of Milli-Qwater for $1.5 \mathrm{~min}$ using UltraTurrex T25 meats homogenizer (Janke and Kenkel, IKA, LaborTecnik, USA).

4- Then an aliquot ( $0.5 \mathrm{~g})$ of homogenized sample was transferred into a glass test tube, fortified with 50 $\mu \mathrm{L}$ of variable concentrations of the working standard solution, leaving the analytes in contact with meat sample for $30 \mathrm{~min}$.

5- After $3 \mathrm{~mL}$ of McIIvaine buffer was added, the mixture was vortexed at high speed, incubated for 5 minutes at room temperature and centrifuged at 3,500 $\mathrm{rpm}$ for $10 \mathrm{~min}$ in a refrigerated centrifuge.

6- The extraction was repeated by adding $2 \mathrm{~mL}$ of McIIvaine buffer and the supernatant was pooled.

7- The supernatant was filtered and loaded on an Oasis HLB6 cm3 (200 mg) polymeric cartridge previously conditioned with $3 \mathrm{~mL}$ of methanol and 2 $\mathrm{mL}$ of water. The cartridge containing the sample was washed with 5 mLof water, and then tetracyclines were eluted with $4.5 \mathrm{~mL}$ of $0.01 \mathrm{M}$ methanolic oxalic acid (pH 1.80).

8- One milliliter of eluent was filtered through 0.22 $\mu \mathrm{m}$ nylon filter, vortexed and centrifuged, and then 20 $\mu \mathrm{L}$ of the aliquot was injected into the HPLC system. 


\section{RESULTS}

Table 1: Minimum, maximum and mean values of oxytetracycline hydrochloride residues in injected rabbits meat $(\mu \mathrm{g} / \mathrm{kg})$ before and after boiling $(\mathrm{n}=5)$.

\begin{tabular}{ccccccc}
\hline & \multicolumn{3}{c}{ Before boiling } & \multicolumn{3}{c}{ After boiling } \\
\cline { 2 - 7 } Statistical analysis & Min. & Max. & $\begin{array}{c}\text { Mean } \\
\pm \text { S.D }\end{array}$ & Min. & Max. & $\begin{array}{c}\text { Mean } \\
\pm \text { S.D }\end{array}$ \\
\cline { 2 - 7 } & 2482.9 & 6235.1 & $4565.1 \pm 1.640$ & 1865.3 & 4356.8 & $2842.1 \pm 0.988$ \\
\hline Zero time & 3398.3 & 4213.5 & $3801.4 \pm 0.341$ & 2227.4 & 4201.2 & $3126.9 \pm 0.885$ \\
\hline After 1 day & 2175.8 & 4325.4 & $3433.2 \pm 0.904$ & 1958.5 & 4215.3 & $3219.4 \pm 0.984$ \\
\hline After 2 days & 2002.5 & 4124.5 & $3238.5 \pm 0.906$ & 1869.5 & 4004.8 & $2845.5 \pm 1.013$ \\
\hline After 3 days & 1854.2 & 3612.4 & $2938.2 \pm 0.755$ & 1625.1 & 3335.3 & $2474.7 \pm 0.665$ \\
\hline After 4 days & 2002.1 & 3400.8 & $2612.1 \pm 0.611$ & 925.4 & 3014.6 & $1568.3 \pm 0.900$ \\
\hline After 5 days & 1432.1 & 2854.1 & $2024.5 \pm 0.559$ & 652.9 & 2425.4 & $1321.1 \pm 0.747$ \\
\hline After 6 days & 896.9 & 2296.5 & $1533.6 \pm 0.636$ & 432.6 & 2072.3 & $1071.9 \pm 0.733$ \\
\hline After 1 week & N.D & 421.6 & $198.2 \pm 0.204$ & N.D & 93.3 & $45.3 \pm 0.042$ \\
\hline After 2 weeks & N.D & 6.8 & $1.4 \pm 0.003$ & N.D & N.D & N.D \\
\hline After 3 weeks & & & & & & \\
\hline
\end{tabular}

Table 2: Minimum, maximum and mean values of oxytetracycline hydrochoride residues in injected rabbits meat $(\mu \mathrm{g} / \mathrm{kg})$ before and after freezing $(\mathrm{n}=5)$.

\begin{tabular}{|c|c|c|c|c|c|c|c|c|c|c|c|c|}
\hline \multirow{2}{*}{ 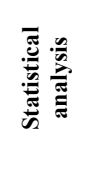 } & \multicolumn{3}{|c|}{ Before freezing } & \multicolumn{3}{|c|}{ After freezing 1 month } & \multicolumn{3}{|c|}{ After freezing 2 month } & \multicolumn{3}{|c|}{ After freezing 3 month } \\
\hline & Min. & Max. & $\begin{array}{c}\text { Mean } \\
\pm \text { S.D }\end{array}$ & Min. & Max. & $\begin{array}{c}\text { Mean } \\
\pm \text { S.D }\end{array}$ & Min. & Max. & $\begin{array}{l}\text { Mean } \\
\pm \text { S.D }\end{array}$ & Min. & Max. & $\begin{array}{l}\text { Mean } \\
\pm \text { S.D }\end{array}$ \\
\hline $\begin{array}{l}\text { Zero } \\
\text { time }\end{array}$ & 2482.9 & 6235.1 & $4565.1 \pm 1.640$ & 2222.4 & 4021.3 & $3370.6 \pm 0.709$ & 1741.1 & 2182.4 & $1950.7 \pm 0.166$ & 421.6 & 1245.8 & $828.6 \pm 0.322$ \\
\hline 1 day & 3398.3 & 4213.5 & $3801.4 \pm 0.341$ & 2387.5 & 4012.8 & $3290.3 \pm 0.769$ & 1532.6 & 2007.2 & $1771.1 \pm 0.198$ & 386.1 & 965.7 & $645.4 \pm 0.223$ \\
\hline 2days & 2175.8 & 4325.4 & $3433.2 \pm 0.904$ & 2014.5 & 4214.9 & $3125.6 \pm 0.946$ & 1333.7 & 1893.5 & $1606.1 \pm 0.220$ & 210.3 & 855.9 & $539.5 \pm 0.244$ \\
\hline 3days & 2002.5 & 4124.5 & $3238.5 \pm 0.906$ & 1924.5 & 4100.3 & $2919.3 \pm 1.016$ & 1144.7 & 1636.6 & $1396.2 \pm 0.174$ & 184.2 & 533.6 & $346.9 \pm 0.147$ \\
\hline 4days & 1854.2 & 3612.4 & $2938.2 \pm 0.755$ & 1754.2 & 3421.8 & $2573.5 \pm 0.651$ & 1055.1 & 1355.5 & $1192.9 \pm 0.114$ & 102.1 & 333.6 & $195.2 \pm 0.089$ \\
\hline 5days & 2002.1 & 3400.8 & $2612.1 \pm 0.611$ & 1125.3 & 2928.4 & $1889.9 \pm 0.667$ & 924.1 & 1163.3 & $1043.8 \pm 0.094$ & 93.4 & 126.5 & $107.4 \pm 0.012$ \\
\hline 6days & 1432.1 & 2854.1 & $2024.5 \pm 0.559$ & 1175.2 & 2632.1 & $1705.9 \pm 0.664$ & 785.1 & 932.2 & $871.3 \pm 0.064$ & 66.2 & 114.6 & $87.3 \pm 0.019$ \\
\hline 1week & 896.9 & 2296.5 & $1533.6 \pm 0.636$ & 514.9 & 2154.7 & $1276.4 \pm 0.322$ & 501.4 & 881.1 & $725.3 \pm 0.150$ & N.D & 112 & $55.4 \pm 0.052$ \\
\hline 2weeks & N.D & 421.6 & $198.2 \pm 0.204$ & N.D & 134.8 & $60.8 \pm 0.059$ & N.D & 8.7 & $1.7 \pm 0.003$ & N.D & N.D & N.D \\
\hline 3weeks & N.D & 6.8 & $1.4 \pm 0.003$ & N.D & N.D & N.D & N.D & N.D & N.D & N.D & N.D & N.D \\
\hline
\end{tabular}


Table 3: Minimum, maximum and mean values of oxytetracycline hydrochloride residues in injected rabbits kidneys $(\mu \mathrm{g} / \mathrm{kg})$ before and after boiling $(\mathrm{n}=5)$.

\begin{tabular}{ccccccc}
\hline \multirow{2}{*}{$\begin{array}{c}\text { Statistical } \\
\text { analysis }\end{array}$} & \multicolumn{3}{c}{ Before boiling } & & After boiling \\
\cline { 2 - 7 } & Min. & Max. & $\begin{array}{c}\text { Mean } \\
\pm \text { S.D }\end{array}$ & Min. & Max. & $\begin{array}{c}\text { Mean } \\
\pm \text { S.D }\end{array}$ \\
\hline Zero time & 21356.4 & 42356.2 & $35355.5 \pm 8.524$ & 9684.2 & 27321.4 & $14626.7 \pm 7.537$ \\
\hline After 1 day & 17852.4 & 28254.1 & $22811.7 \pm 4.275$ & 7128.6 & 16589.2 & $10245.1 \pm 4.137$ \\
\hline After 2 days & 12182.3 & 20398.2 & $15329.5 \pm 3.619$ & 4827.3 & 11324.1 & $7217.3 \pm 2.944$ \\
\hline After 3 days & 7928.3 & 18925.1 & $10929.2 \pm 4.680$ & 5006.3 & 13842.1 & $7979.9 \pm 3.484$ \\
\hline After 4 days & 4625.1 & 12653.2 & $7409.4 \pm 3.579$ & 3018.6 & 9897.2 & $5294.6 \pm 3.059$ \\
\hline After 5 days & 3483.7 & 9653.4 & $5872.9 \pm 2.677$ & 2016 & 6591.6 & $3819.6 \pm 1.866$ \\
\hline After 6 days & 3521.3 & 8321.4 & $5247 \pm 2.011$ & 1834.6 & 5914.6 & $3201 \pm 1.712$ \\
\hline After 1 week & 1632.5 & 3872.5 & $2540 \pm 0.894$ & 593.4 & 2095.1 & $1155.5 \pm 0.620$ \\
\hline After 2 weeks & 264.8 & 1653.4 & $965.1 \pm 0.543$ & 14.7 & 923.7 & $510 \pm 0.364$ \\
\hline After 3 weeks & N.D & 32.5 & $17.7 \pm 0.014$ & N.D & N.D & N.D \\
\hline
\end{tabular}

Table 4: Minimum, maximum and mean values of oxytetracycline hydrochloride residues in injected rabbits kidneys $(\mu \mathrm{g} / \mathrm{kg})$ before and after freezing $(\mathrm{n}=5)$.

\begin{tabular}{|c|c|c|c|c|c|c|c|c|c|c|c|c|}
\hline \multirow{2}{*}{ 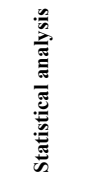 } & \multicolumn{3}{|c|}{ Before freezing } & \multicolumn{3}{|c|}{ After freezing 1 month } & \multicolumn{3}{|c|}{ After freezing 2 month } & \multicolumn{3}{|c|}{ After freezing 3 month } \\
\hline & Min. & Max. & $\begin{array}{l}\text { Mean } \\
\pm \text { S.D }\end{array}$ & Min. & Max. & $\begin{array}{l}\text { Mean } \\
\pm \text { S.D }\end{array}$ & Min. & Max. & $\begin{array}{l}\text { Mean } \\
\pm \text { S.D }\end{array}$ & Min. & Max. & $\begin{array}{l}\text { Mean } \\
\pm \text { S.D }\end{array}$ \\
\hline $\begin{array}{l}\text { Zero } \\
\text { time }\end{array}$ & 21356.4 & 42356.2 & $35355.5 \pm 8.524$ & 14215.6 & 36215.4 & $26964.3 \pm 8.531$ & 4623.3 & 6229.3 & $5297.8 \pm 0.614$ & 1339.4 & 2365.1 & $1834.3 \pm 0.416$ \\
\hline 1 day & 17852.4 & 28254.1 & $22811.7 \pm 4.275$ & 12549.7 & 21321.5 & $17324.9 \pm 3.796$ & 3499.9 & 5277.1 & $4196.7 \pm 0.675$ & 1047.2 & 1967.7 & $1482.6 \pm 0.386$ \\
\hline 2days & 12182.3 & 20398.2 & $15329.5 \pm 3.619$ & 9231.7 & 14328.1 & $10865.6 \pm 2.095$ & 2901.3 & 3331.8 & $3077.2 \pm 0.162$ & 1036.4 & 1756.3 & $1271.6 \pm 0.294$ \\
\hline 3days & 7928.3 & 18925.1 & $10929.2 \pm 4.680$ & 7010.7 & 14012.8 & $9108.7 \pm 2.812$ & 2110.1 & 3185.7 & $2806.7 \pm 0.414$ & 823.4 & 1389.1 & $1074.5 \pm 0.216$ \\
\hline 4days & 4625.1 & 12653.2 & $7409.4 \pm 3.579$ & 3392.1 & 11926.1 & $6242.7 \pm 3.962$ & 1441.8 & 2314.4 & $1882 \pm 0.318$ & 585.7 & 1330.1 & $911.7 \pm 0.308$ \\
\hline 5days & 3483.7 & 9653.4 & $5872.9 \pm 2.677$ & 2518.8 & 7325.2 & $4428.4 \pm 2.147$ & 1263.6 & 2132.1 & $1696.6 \pm 0.377$ & 503.3 & 963.1 & $767.8 \pm 0.199$ \\
\hline 6days & 3521.3 & 8321.4 & $5247 \pm 2.011$ & 2368.2 & 6821.4 & $4088.4 \pm 1.894$ & 1085.8 & 1788.9 & $1384.5 \pm 0.308$ & 239.4 & 634.4 & $466.3 \pm 0.152$ \\
\hline 1week & 1632.5 & 3872.5 & $2540 \pm 0.894$ & 1186.3 & 3102.8 & $1927.6 \pm 0.769$ & 322.7 & 1232.6 & $701.7 \pm 0.434$ & 62.4 & 322.7 & $187.1 \pm 0.118$ \\
\hline 2weeks & 264.8 & 1653.4 & $965.1 \pm 0.543$ & 28.4 & 1359.1 & $720 \pm 0.516$ & N.D & 52.1 & $20.3 \pm 0.021$ & N.D & 11.7 & $5 \pm 0.005$ \\
\hline 3weeks & N.D & 32.5 & $17.7 \pm 0.014$ & N.D & N.D & N.D & N.D & N.D & N.D & N.D & N.D & N.D \\
\hline
\end{tabular}


Assiut Vet. Med. J. Vol. 60 No. 141 April 2014

Table 5: Minimum, maximum and mean values of oxytetracycline hydrochloride residues in injected rabbits livers $(\mu \mathrm{g} / \mathrm{kg})$ before and after boiling $(\mathrm{n}=5)$.

\begin{tabular}{ccccccc}
\hline & \multicolumn{3}{c}{ Before boiling } & \multicolumn{3}{c}{ After boiling } \\
\cline { 2 - 7 } Statistical analysis & Min. & Max. & $\begin{array}{c}\text { Mean } \\
\pm \text { S.D }\end{array}$ & Min. & Max. & $\begin{array}{c}\text { Mean } \\
\pm \text { S.D }\end{array}$ \\
\cline { 2 - 7 } & 5985.4 & 10238.1 & $7393.1 \pm 1.832$ & 3751.4 & 7392.7 & $5196.9 \pm 1.781$ \\
\hline Zero time & 4724.4 & 8963.2 & $6475.6 \pm 1.766$ & 3009.7 & 6492.8 & $4418.1 \pm 1.449$ \\
\hline After 1 day & 3734.8 & 6362.8 & $4838.5 \pm 1.216$ & 2821.7 & 4893.4 & $3454.9 \pm 0.834$ \\
\hline After 2 days & 3093.4 & 5932.4 & $4111.2 \pm 1.147$ & 2494.7 & 4003.4 & $3223.4 \pm 0.598$ \\
\hline After 3 days & 2892.1 & 4682.9 & $3626.5 \pm 0.755$ & 2005.4 & 3834.7 & $2821.9 \pm 0.781$ \\
\hline After 4 days & 2632.6 & 4321.4 & $3321.3 \pm 0.671$ & 1870.8 & 3591.7 & $2615.6 \pm 0.662$ \\
\hline After 5 days & 2074.5 & 3745.2 & $2962.2 \pm 0.642$ & 1558.4 & 2931.2 & $2164.4 \pm 0.525$ \\
\hline After 6 days & 1636.3 & 3112.5 & $2609.8 \pm 0.624$ & 800.7 & 2451.3 & $1538.4 \pm 0.713$ \\
\hline After 1 week & 472.6 & 1421.8 & $1037.6 \pm 0.422$ & 63.4 & 733 & $481.7 \pm 0.267$ \\
\hline After 2 weeks & N.D & 14.2 & $3.8 \pm 0.006$ & N.D & N.D & N.D \\
\hline After 3 weeks & & & & & & \\
\hline & & & & &
\end{tabular}

Table 6: Minimum, maximum and mean values of oxytetracycline hydrochloride residues in injected rabbits livers $(\mu \mathrm{g} / \mathrm{kg})$ before and after freezing $(\mathrm{n}=5)$.

\begin{tabular}{|c|c|c|c|c|c|c|c|c|c|c|c|c|}
\hline \multirow{2}{*}{ 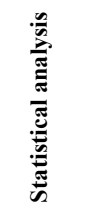 } & \multicolumn{3}{|c|}{ Before freezing } & \multicolumn{3}{|c|}{ After freezing 1 month } & \multicolumn{3}{|c|}{ After freezing 2 month } & \multicolumn{3}{|c|}{ After freezing 3 month } \\
\hline & Min. & Max. & $\begin{array}{c}\text { Mean } \\
\pm \text { S.D }\end{array}$ & Min. & Max. & $\begin{array}{c}\text { Mean } \\
\pm \text { S.D }\end{array}$ & Min. & Max. & $\begin{array}{c}\text { Mean } \\
\pm \text { S.D }\end{array}$ & Min. & Max. & $\begin{array}{c}\text { Mean } \\
\pm \text { S.D }\end{array}$ \\
\hline $\begin{array}{l}\text { Zero } \\
\text { time }\end{array}$ & 5985.4 & 10238.1 & $7393.1 \pm 1.832$ & 4782.9 & 9543.8 & $6545.5 \pm 2.021$ & 3869.6 & 7213.5 & $5512.9 \pm 1.507$ & 2614.6 & 4823.4 & $3328.5 \pm 0.875$ \\
\hline 1 day & 4724.4 & 8963.2 & $6475.6 \pm 1.766$ & 4392.5 & 7654.8 & $5949.1 \pm 1.327$ & 3596.1 & 6366.6 & $4978.9 \pm 1.161$ & 2300.7 & 4621.4 & $3112.7 \pm 0.909$ \\
\hline 2days & 3734.8 & 6362.8 & $4838.5 \pm 1.216$ & 3293.8 & 5721.8 & $4196.4 \pm 1.052$ & 3187.3 & 5236.8 & $4353.6 \pm 0.849$ & 2401.7 & 4321.5 & $2959.9 \pm 0.799$ \\
\hline 3days & 3093.4 & 5932.4 & $4111.2 \pm 1.147$ & 2814.9 & 5020.7 & $3637.3 \pm 0.874$ & 2711.7 & 4917.4 & $3532.2 \pm 0.881$ & 2187.9 & 3621.4 & $2581.5 \pm 0.588$ \\
\hline 4days & 2892.1 & 4682.9 & $3626.5 \pm 0.755$ & 2563.8 & 4324.9 & $3356.7 \pm 0.314$ & 2222.1 & 4236.3 & $3096.9 \pm 0.822$ & 1766.2 & 2824.5 & $2122.8 \pm 0.420$ \\
\hline 5days & 2632.6 & 4321.4 & $3321.3 \pm 0.671$ & 2111.9 & 3982.4 & $2918.6 \pm 0.715$ & 2086.7 & 3775.2 & $2725.9 \pm 0.711$ & 1448.1 & 2633 & $1908.7 \pm 0.451$ \\
\hline 6days & 2074.5 & 3745.2 & $2962.2 \pm 0.642$ & 1845.2 & 3222.3 & $2408.3 \pm 0.565$ & 1735.1 & 3030.2 & $2261.6 \pm 0.518$ & 1085.1 & 1845.5 & $1441.4 \pm 0.303$ \\
\hline 1week & 1636.3 & 3112.5 & $2609.8 \pm 0.624$ & 1184.7 & 2884.9 & $2157.7 \pm 0.655$ & 1096.3 & 2785.4 & $1942.6 \pm 0.648$ & 239.5 & 1366.9 & $719.1 \pm 0.474$ \\
\hline 2weeks & 472.6 & 1421.8 & $1037.6 \pm 0.422$ & 294.5 & 1293.1 & $899.8 \pm 0.437$ & N.D & 1120.1 & $433 \pm 0.559$ & N.D & 793.3 & $262.9 \pm 0.372$ \\
\hline 3weeks & N.D & 14.2 & $3.8 \pm 0.006$ & N.D & N.D & N.D & N.D & N.D & N.D & N.D & N.D & N.D \\
\hline
\end{tabular}

Min. $=$ Minimum, Max. $=$ Maximum, N.D $=$ Not detected and S.D $=$ Standard Deviation 
Figure1: HPLC chromatogram of meat sample of the group slaughtered at zero time after the last dose of oxytetracycline $(6235.1 \mu \mathrm{g} / \mathrm{kg})$.

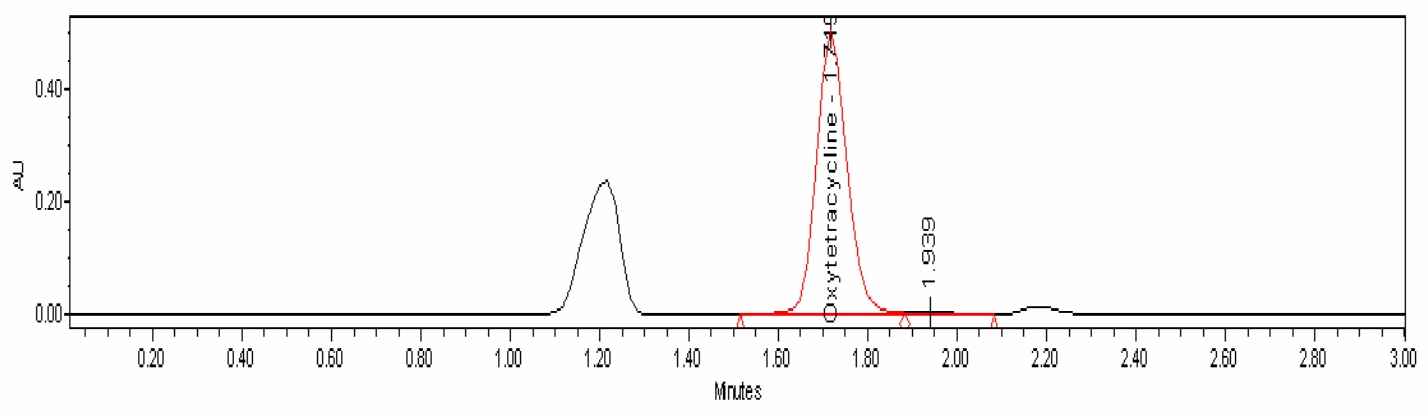

Figure 2: HPLC chromatogram of meat sample of the group slaughtered one weeks after the Last dose of oxytetracycline and freezed for three months $(87.5 \mu \mathrm{g} / \mathrm{kg})$.

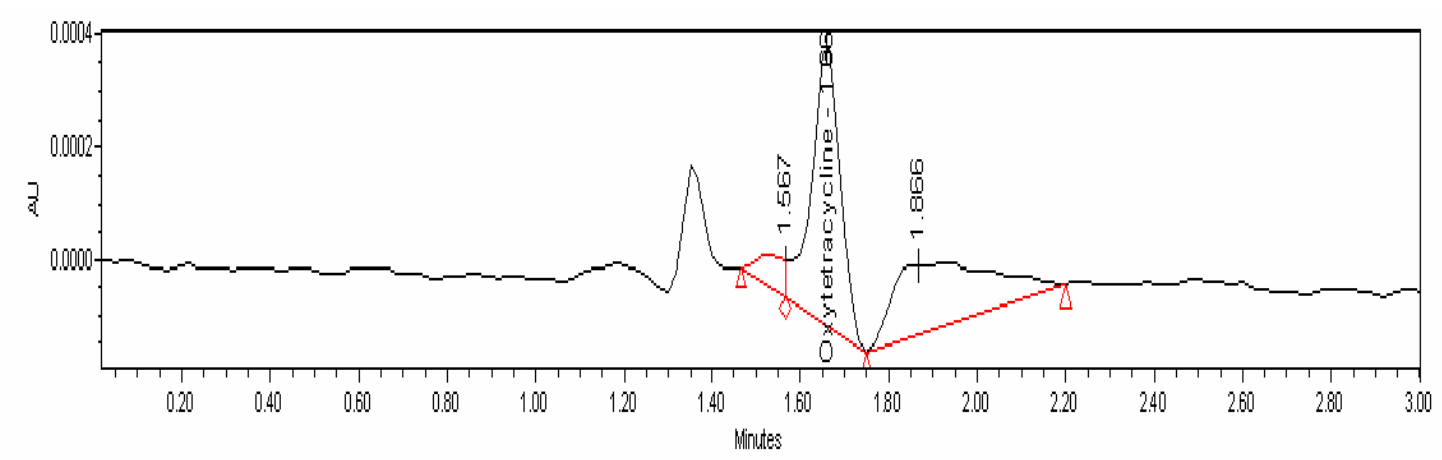

Figure 3: HPLC chromatogram of kidney sample of the group slaughtered two weeks after the Last dose of oxytetracycline and freezed for one month $(438.2 \mu \mathrm{g} / \mathrm{kg})$.

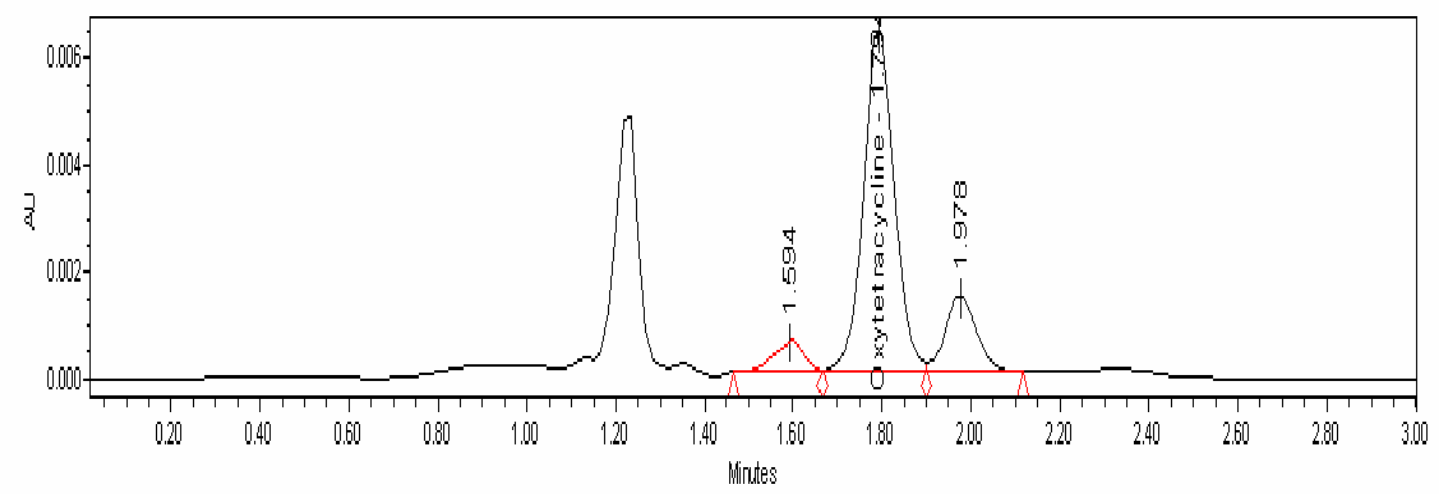




\section{DISCUSSION}

The obtained results in table (1) showed that the mean values of oxytetracycline in meat after slaughter time zero, one day, two days, three days, four days, five days, six days, one week, two weeks and three weeks from the last dose $(20 \mathrm{mg} / \mathrm{kg}$ body weight for 5 successive days) estimated by HPLC was $4565.1 \pm 1.640, \quad 3801.4 \pm 0.341, \quad 3433.2 \pm 0.904$, $3238.5 \pm 0.906, \quad 2938.2 \pm 0.755, \quad 2612.1 \pm 0.611$, $2024.5 \pm 0.559, \quad 1533.6 \pm 0.636, \quad 198.2 \pm 0.204$ and $1.4 \pm 0.003 \mu \mathrm{g} / \mathrm{kg}$, respectively, but after boiling they were $2842.1 \pm 0.988, \quad 3126.9 \pm 0.885, \quad 3219.4 \pm 0.984$, $2845.5 \pm 1.013, \quad 2474.7 \pm 0.665, \quad 1568.3 \pm 0.900$, 1321.1 $\pm 0.747, \quad 1071.9 \pm 0.733, \quad 45.3 \pm 0.042$ and undetected $\mu \mathrm{g} / \mathrm{kg}$, respectively, while after freezing results in table (2) showed that for one month they were $3370.6 \pm 0.709, \quad 3290.3 \pm 0.769,3125.6 \pm 0.946$, $2919.3 \pm 1.016, \quad 2573.5 \pm 0.651, \quad 1889.9 \pm 0.667$, $1705.9 \pm 0.664, \quad 1276.4 \pm 0.322, \quad 60.8 \pm 0.059$ and undetected $\mu \mathrm{g} / \mathrm{kg}$, respectively, after freezing for two months they were $1950.7 \pm 0.166,1771.1 \pm 0.198$, $1606.1 \pm 0.220, \quad 1396.2 \pm 0.174, \quad 1192.9 \pm 0.114$, $1043.8 \pm 0.094,871.3 \pm 0.064,725.3 \pm 0.150,1.7 \pm 0.003$ and undetected $\mu \mathrm{g} / \mathrm{kg}$, respectively and after freezing for three months they were $828.6 \pm 0.322,645.4 \pm 0.223$, $539.5 \pm 0.244,346.9 \pm 0.147,195.2 \pm 0.089,107.4 \pm 0.012$ $87.3 \pm 0.019,55.4 \pm 0.052$, undetected and undetected $\mu \mathrm{g} / \mathrm{kg}$, respectively. It is clearly appeared that the time of slaughter after the last injected dose of oxytetracycline significantly affects the concentration of oxytetracycline in muscle, as slaughtering of rabbits after prolonged time of last dose of oxytetracycline decreased greatly the residual level until the concentration became very low at slaughter time 3 weeks after the last dose of oxytetracycline. These results are in agreement approximately with Rome (1991) and Villa et al. (2001) who concln.ded that there were significant differences among the sacrifice times $(0,24,48$ and 72 hours post- treatment) in all matrixes collected in rabbits and by increasing the slaughter time after the last dose, the oxytetracycline concentration decreases. Boiling significantly decreases oxytetracycline level in muscle till complete disappearance of oxytetracycline at slaughter time 3 weeks after the last dose of oxytetracycline. Oxytetracycline residues decreased by boiling (Nashwa, 2012). Cooking significantly affect the oxytetracycline level in turkey meat (Javedi, 2011). These results were nearly similar to previously recorded results Javedi (2011) and Nashwa (2012) and disagreed with those recorded by Mohammed (1997). Freezing caused partial degradation of oxytetracycline in relation to the slaughter time after the last dose injected and the period of freezing, as long storage of rabbit muscles at freezing temperature $\left(-20^{\circ} \mathrm{c}\right)$ decreased the oxyteracycline residues until disappeared completely from the rabbit muscles which slaughtered 2 weeks and 3 weeks after the last dose injected and stored at $-20^{\circ} \mathrm{c}$ for 3 months. These results were in a partial agreement with Hagsmaa (1993), Kan (1995), Mansour (2000), Abd El-Monem et al. (2002), Gehad (2002), Hanaa (2002) and Tamer (2012), as they concluded that tetracycline residues were degraded by freezing at $-18^{\circ} \mathrm{c}$ to $-20^{\circ} \mathrm{c}$. While these results were disagreed with those obtained by Gehan (1991).

Table (3) showed that the mean values of oxytetracycline in kidney samples after slaughter time zero, one day, two days, three days, four days, five days, six days, one week, two weeks and three weeks was $35355.5 \pm 8.524,22811.7 \pm 4.275,15329.5 \pm 3.619$, $10929.2 \pm 4.680, \quad 7409.4 \pm 3.579, \quad 5872.9 \pm 2.677$, $5247 \pm 2.011, \quad 2540 \pm 0.894, \quad 965.1 \pm 0.543 \quad$ and $17.7 \pm 0.014 \mu \mathrm{g} / \mathrm{kg}$, respectively, while after boiling the mean values of oxytetracycline was $14626.7 \pm 7.537$, 10245.1 $\pm 4.137, \quad 7217.3 \pm 2.944, \quad 7979.9 \pm 3.484$, $5294.6 \pm 3.059, \quad 3819.6 \pm 1.866, \quad 3201 \pm 1.712$, $1155.5 \pm 0.620, \quad 510 \pm 0.364$ and undetected $\mu \mathrm{g} / \mathrm{kg}$, respectively, and table(4) showed that after freezing for one month the mean values of oxytetracycline was $26964.3 \pm 8.531,17324.9 \pm 3.796,10865.6 \pm 2.095$, $9108.7 \pm 2.812, \quad 6242.7 \pm 3.962, \quad 4428.4 \pm 2.147$, $4088.4 \pm 1.894, \quad 1927.6 \pm 0.769, \quad 720 \pm 0.516 \quad$ and undetected $\mu \mathrm{g} / \mathrm{kg}$, respectively, after freezing for two months the mean values was $5297.8 \pm 0.614$, $4196.7 \pm 0.675, \quad 3077.2 \pm 0.162, \quad 2806.7 \pm 0.414$, $1882 \pm 0.318, \quad 1696.6 \pm 0.377, \quad 1384.5 \pm 0.308$, $701.7 \pm 0.434, \quad 20.3 \pm 0.021$ and undetected $\mu \mathrm{g} / \mathrm{kg}$, respectively and after freezing for three months they were $1834.3 \pm 0.416, \quad 1482.6 \pm 0.386, \quad 1271.6 \pm 0.294$, $1074.5 \pm 0.216, \quad 911.7 \pm 0.308, \quad 767.8 \pm 0.199$, $466.3 \pm 0.152,187.1 \pm 0.118,5 \pm 0.005$ and undetected $\mu \mathrm{g} / \mathrm{kg}$, respectively. High oxytetracycline concentrations were detected in the kidney and this is due to the kidney is the route of excretion of oxytetracycline. The level of oxytetracycline decreased with the increase of slaughter time. Nearly similar results were reported by Rome (1991) and Villa et al. (2001). Data illustrated in table (5) showed that the mean values of oxytetracycline after slaughter time at zero, one day, two days, three days, four days, five days, six days, one week, two weeks and three weeks was $7393.1 \pm 1.832,6475.6 \pm 1.766$, $4838.5 \pm 1.216, \quad 4111.2 \pm 1.147, \quad 3626.5 \pm 0.755$, $3321.3 \pm 0.671, \quad 2962.2 \pm 0.642, \quad 2609.8 \pm 0.624$, $1037.6 \pm 0.422$ and $3.8 \pm 0.006 \mu \mathrm{g} / \mathrm{kg}$, respectively, while after boiling they were 5196.9 \pm 1.781 , $4418.1 \pm 1.449, \quad 3454.9 \pm 0.834, \quad 3223.4 \pm 0.598$, $2821.9 \pm 0.781, \quad 2615.6 \pm 0.662, \quad 2164.4 \pm 0.525$, $1538.4 \pm 0.713,481.7 \pm 0.267$ and undetected $\mu \mathrm{g} / \mathrm{kg}$, respectively, and as showed in table (6) after freezing for one month they were $6545.5 \pm 2.021,5949.1 \pm 1.327$, $4196.4 \pm 1.052, \quad 3637.3 \pm 0.874, \quad 3356.7 \pm 0.314$, $2918.6 \pm 0.715, \quad 2408.3 \pm 0.565, \quad 2157.7 \pm 0.655$, $899.8 \pm 0.437$ and undetected $\mu \mathrm{g} / \mathrm{kg}$, respectively, after freezing for two months they were 5512.9 \pm 1.507 , $4978.9 \pm 1.161, \quad 4353.6 \pm 0.849, \quad 3532.2 \pm 0.881$, $3096.9 \pm 0.822, \quad 2725.9 \pm 0.711, \quad 2261.6 \pm 0.518$, 
$1942.6 \pm 0.648, \quad 433 \pm 0.559$ and undetected $\mu \mathrm{g} / \mathrm{kg}$, respectively and after freezing for three months they were $3328.5 \pm 0.875, \quad 3112.7 \pm 0.909, \quad 2959.9 \pm 0.799$, $2581.5 \pm 0.588, \quad 2122.8 \pm 0.420, \quad 1908.7 \pm 0.451$, $1441.4 \pm 0.303, \quad 719.1 \pm 0.474, \quad 262.9 \pm 0.372 \quad$ and undetected $\mu \mathrm{g} / \mathrm{kg}$, respectively. The liver is the site of metabolism of oxytetracycline so the level of residual oxytetracycline is high in liver and begin gradually to be decreased with increasing the slaughter time after the last dose till reached a very low concentration after slaughter time 3 weeks. These results were nearly similar to those obtained by Rome (1991) and Villa et al. (2001). These results disagreed with those recorded by Mohammed (1997), Hanaa (2002) and Tamer (2012) that clarified that oxytetracycline completely disappeared by boiling regardless the initial concentration before boiling.

The recovery rates for oxytetracycline in rabbit meat, kidneys and livers was $102 \%, 92 \%$ and $86 \%$, respectively, at concentration of $20 \mu \mathrm{g} / \mathrm{kg}$ of spiked samples.

It can be concluded that neither boiling nor freezing could be considered as a reliable method to get rid completely from oxytetracycline as they caused a partial degradation of oxytetracycline residues. The only solution depending on the obtained results is to delay the slaughter time till the oxytetracycline metabolized in liver and excreted from the kidneys, and this takes a withdrawal period about 3 weeks after a course of treatment $20 \mathrm{mg} / \mathrm{kg}$ body weight subcutaneously for five successive days.

\section{REFERENCES}

Abd El-Monem, K.M.; Soliman, M.R. and Saad, S.M. (2002): Oxytetracycline residues in broiler carcasses produced by closed and open system. J. Egypt Vet. Med. Ass., 62(6a): 119-124.

Badiola, J.I.; González, J.; Aloy, N. and Pérez de Rozas, A.M. (2007): El uso de antimicrobianos en granjas cunícolas: necesidades de registro, uso prn. dente y medidas alternativas. In Proc. II Congreso Ibérico de Cunicultura, 2007 May, Vila-Real ، Portugal, 173-181.

BiswAs, A.K.; RAo, G.s.; KoNdAiAh, N.; ANjANeyulu, A.S.R.; MeNdiRAttA, S.K.; PRASAd, R. and MAliK, J.K. (2007): A Simple Multiresidue Method for Determination of Oxytetracycline, Tetracycline and Chlortetracycline in Export Buffalo Meat by HPLC-Photodiode Array Detector. Journal of Food and Drug Analysis, Vol. 15, No. 3, 2007, Pages 278-284.

Boisseau, J. (1993): Basis for the evaluation of the microbiological risks due to veterinary drug residues in food. Vet. Microbiol. 35, 187-192.
Gehad, F.A. (2002): Stability of some Veterinary Drug Residues in animal meats, During storage, Preparation and Processing. Ph.D.V.Sc. Thesis Fac. Vet. Med.

Gehan, R.M. (1991): Studies on the withdrawal time of some antibiotics from broilers and rabbits. M.V.Sc. Thesis Fac. Vet. Med. Zagazig University, Moshtohor Branch.

Hagsmaa, N. (1993): Stability of veterinary drug residues during storage and processing. Proceeding of the European Residue conference, Veldhoren, Netherland, 1:41-48.

Hanaa, M.M. Sultan (2002): Residual status of some growth promoters and chemotherapeutics in meat and offals of slaughtered cattle and sheep. Ph.D.V.Sc. Dept. of food hygiene and control, Cairo University.

Javadi, A. (2011): Effect of roasting, boiling and microwaving cooking method on Doxycline residues in edible meats of poultry by microbial method. African Journal of Pharmacy and Pharmacology Vol. 5(8), pp. 1034-1037.

Kan, C.A. (1995): Residues probability and their occurrence and efficiency of their detection. Misset- World poultry J. 11(10): 67-73.

Mansour, A.H.M. (2000): Stn.dies on antibiotic residues in turkey meat and offal. Ph. D. Thesis, Dept. of food control, Faculty of Vet. Medicine, Moshtohor, Zagazig univ., Benha branch, Egypt.

Mehran Mesgari Abasi; Mohammad Reza Rashidi; Afshin Javadi; Maryam Bannazadeh Amirkhiz; Solmaz Mirmahdavi and Maryam Zabihi. (2009): Levels of tetracycline residues in cattle meat, liver, and kidney from a slaughterhouse in Tabriz, Iran. Turk. J. Vet. Anim. Sci. 2009; 33(4): 345-349.

Moats, W.A. (1997): Advances in determination of antibiotic residues. Journal of AOAC international, $80,1 \pm 4$.

Mohammed, H.I. (1997): Antibiotic residues in rabbit carcasses with special reference to the effect of some processing on its stability. M.V.Sc. Thesis, Fac. Vet. Med.

Nashwa, M.Z. (2012): Seasonal variation of antibiotic residues in some slaughtered animal. Ph.D. of Vet. Med. Sciences, Dept. of food control, Zagazig University.

Rome, A. (1991): Residues of some veterinary drugs in animals and food. FAO / Food and Nutrition papers, 97-119.

Tamer, M.A.G. (2012): Effect of age and sex of animal on the antibiotic residues in meats of some slaughtered animals. Ph.D.V.Sc., food control dept., Zagazig University.

Teske, R. (1993): Microbiological significance of drug residues in food welcome and introduction. Vet. Hum. Toxicol. 35, 1-2. 
Villa, R.; Cagnardi, P.; Bacchetta, S.; Sonzogni, O.; Faustini, M. and Carli, S. (2001): Meat distripution and residue depletion of oxytetracycline in the rabbit. World rabbit Science 2001, vol. 9(4), 159-164.
Yu, H; Tao, Y.; Chen, D.; Wang, Y. and Yuan, Z. (2011): Development of an HPLC-UV method for the simultaneous determination of tetracyclines in muscle and liver of porcine, chicken and bovine with accelerated solvent extraction. Food Chem. 124: 1131-1138.

\section{دراسات عن نضوب بقايا الأوكسي تتراسيكلين في لحوم الأرانب \\ علاء اللدين محمد مرشدي ، محمدعب الله حسين ، عمرو السبد الجوهري \\ Email: elged2010@yahoo.com}

تعتبر لحوم الأر انب منتج عالي الجودة لاحتو ائه على نسبة بروتين عالية ونسبة قليلة من الحموض الدهنية الدئية المشبعة والكوليسترول

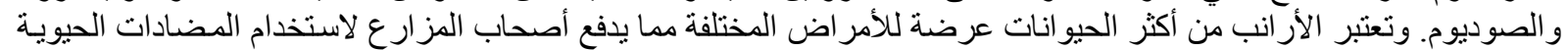

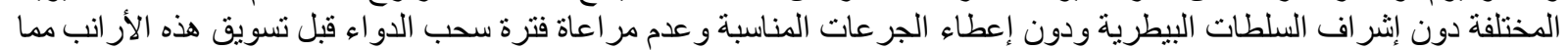

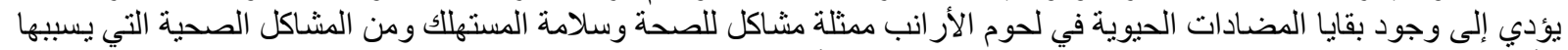

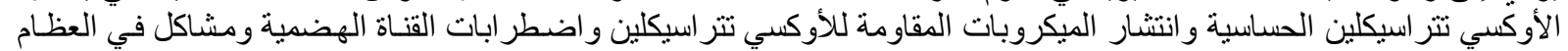

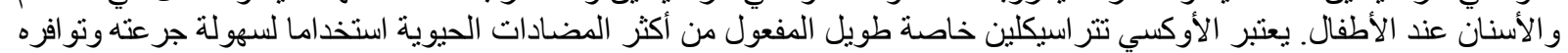

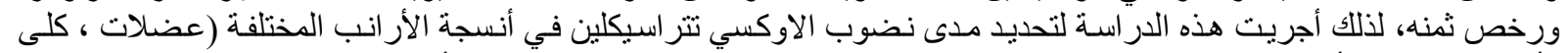

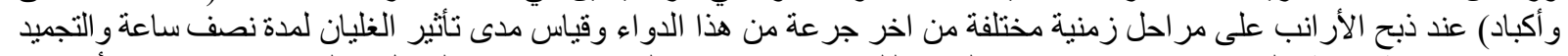

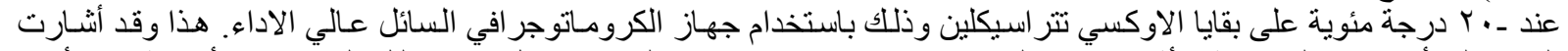

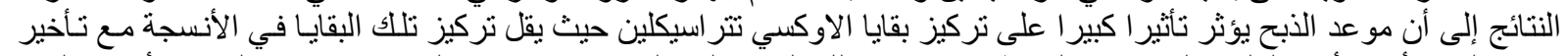

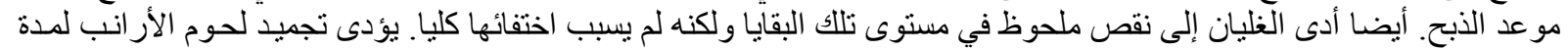

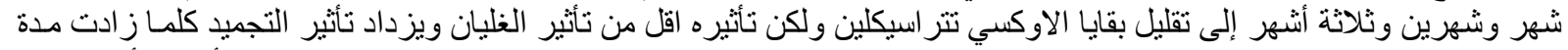

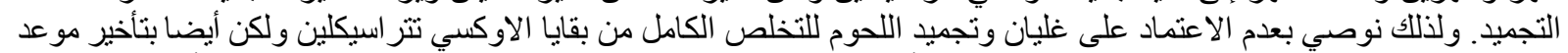
الذبح و الالتز ام بفترة سحب الدو اء و هي في حدود ثلاثة أسابيع للتخلص التنام من بقايا الاوكسي نتز اسيكلين في لحوم الأر انب. 